\title{
Managing bleeding complications in patients treated with the old and the new anticoagulants.
}

\author{
Romualdi E, Rancan E, Siragusa S, $\underline{\text { Ageno W. }}$
}

Source

Research Center on Thromboembolic Disorders and on Antithrombotic Therapies, Department of Clinical Medicine, University of Insubria, Varese, Italy.

\section{Abstract}

An increasing number of patients receive anticoagulant therapy to prevent and treat arterial or venous thromboembolism. The major complication of anticoagulant therapy is the increase of the individual bleeding risk. All anticoagulant drugs can cause haemorrhages, that can sometimes be life-threatening. Although heparins and the vitamin $\mathrm{K}$ antagonists have been the most widely used anticoagulants for decades, the correct management of bleeding complications associated with these agents has been poorly studied. More recently, new anticoagulant drugs, both parenteral and oral, have been approved for clinical use. Currently, none of these new agents has a specific antidote, and little advise can be given on how to manage a major bleeding event. The aim of this article is to describe the haemorrhagic risk and the management of bleeding complications associated with the principal anticoagulant drugs 